\title{
High specificity of spectral and power Doppler ultrasonographic nail assessment in psoriatic arthritis patients
}

\author{
José Alexandre Mendonça ${ }^{1}$, Vânia Aparecida Leandro-Merhi ${ }^{1,2}$, and José Luis Braga de \\ Aquino ${ }^{1}$ \\ ${ }^{1}$ PUC-Campinas \\ ${ }^{2}$ Pontifícia Universidade Católica de Campinas
}

July 23, 2020

\begin{abstract}
Introduction: The use of high resolution ultrasonography (US) became a very important tool in nail assessment. This study evaluated nails clinical and ultrasound measurements in the psoriatic arthritis (PsA) patients and control groups. Methods: A cross sectional study was performed and 44 patients with PsA, 10 health individuals and 6 hand osteoarthritis patients (OA) were selected. PsA patients were included when filling the CASPAR criteria. Results: spectral Doppler (sD) was analyzed in 208 nail beds. Nail plates appearance on gray scale (GS) showed loss of the normal three-layered appearance in 89 nails (45.9\%). Power Doppler (PD) signal was detected in 189 nail beds (92.2\%), showing inflammatory activity in most of the patients and nails examined. Resistance index (RI) measured was significantly lower in PsA patients as compared with controls group in both longitudinal and transverse planes $(\mathrm{p}<0.001)$. The nail enthesitis was observed when $\mathrm{RI}$ values below 0.4 , characterizing $100 \%$ sensitivity and $96 \%$ specificity $(\mathrm{p}<0.01)$. Conclusions: Nail US measurements presented statistical significance in PsA patients, in studies future could show many inflammatory situations, as treatment assessment.
\end{abstract}

\section{Introduction}

PsA is a joint inflammatory disease with both peripheral and axial manifestations. It has been recognized that enthesitis is an important lesion and a multifaceted skeletal manifestations in this disease ${ }^{1}$. Some studies have shown enthesopathy in asymptomatic large insertions of the lower limbs in patients with spondyloarthropathies including $\mathrm{PsA}^{2}$. Several studies have demonstrated subclinical enthesopathy or osteitis in as many as $50 \%$ of psoriasis patients with no skeletal symptoms ${ }^{3}$.

Nail plate involvement is an extremely common feature of psoriasis and affects approximately $10 \%$ to $78 \%$ of psoriasis patients. Nail anatomic structure has been recognized as an enthesis targeted in the inflammatory process of PsA. Different studies have shown that up to $30 \%$ of patients with psoriasis have PsA of which $70 \%-80 \%$ have nail changes ${ }^{4,5}$. A patient with nail psoriasis should be screened for early signs and symptoms of PsA in order to avoid progressive joint damage ${ }^{6}$.

US has been increasingly used for evaluation of joint diseases. It has been found to be as good as magnetic resonance imaging in detection of synovitis ${ }^{7}$. Advantages of US include noninvasiveness, safety, low cost, lack of radiation exposure, and high patient acceptability; enables a rapid high-resolution soft tissue examination and the ability to scan multiple joints at a time. The high resolution US technology is compound from GS, $\mathrm{PD}$ and sD. This technological tool have been used and validate in assessing disease activity in rheumatoid arthritis $^{8-10}$. The parameters PD and sD advantages are that these can estimate both high as well as low velocity blood flow quantitatively. PD to some extent provides a reflection of the degree of angiogenesis, which is critically related to joint damage and therapeutic responses to drugs ${ }^{11,12}$. Extra-synovial PD signal has been demonstrated in both PsA and psoriasis patients as in entheses and paratendon ${ }^{13-16}$. So, 
the RI is a quantitative indice that have been used to evaluate tissue abnormal blood flow in inflammatory arthropathy ${ }^{17}$.

The high-resolution US evaluation has been revealed a perspective important in the PsA joint lesions. ${ }^{18-20}$. There are many nail findings to be discovered by ultrasound, mainly in relation to sD. A previous study has demonstrated that patients with psoriasis have lower blood flow in the nail fold compared with healthy control group $^{21}$. In this present study evaluated sD nail findings in PsA patients as compared with healthy individuals and patients with hand OA. Our hypothesis was that sD nail findings could help detect inflammatory process active in PsA patients.

\section{Methods}

The study was perfromed at the Instituto de Pesquisa Clínica de Campinas - IPECC and Pontifícia Universidade Católica Campinas at both Rheumatology and Dermatology Divisions. Patients were seen as routine outpatients at the Rheumatology and Dermatology Divisions and had US evaluation as an extension of their physical examination. Ethical approval was obtained before starting the study protocol, opinion number: 1.526.307. The nail bed assessment to the $\mathrm{sD}$, semiquantitative GS and PD scores were randomly selected without aleatorization because there weren't any prior standards in the literature.

Patient groups and clinical assessment

A total of 44 consecutive PsA patients, 10 healthy individuals and 6 patients with hand OA were included. PsA patients were included if they fulfilled the CASPAR criteria ${ }^{22}$. Clinical assessment was performed by a rheumatologist and a dermatologist who were blinded to the ultrasound findings. Patients were seen for clinical evaluation before US examination and the following disease-related parameters were evaluated: age, sex and ethnic group, psoriasis area and severity index (PASI), patient's global pain and nail pain (each 0 to $100 \mathrm{~mm}$ visual analogue scale for global pain -VASGp and visual analogue scale for nail pain VASNp, respectively), and disease and treatment duration. Specific medication use was also recorded for all patients.

The US examinations of musculoskeletal system were performed with multiplanar technique, at the clinically involved sites adopting the indications provided by the European League Against Rheumatism guidelines for musculoskeletal ultrasound in rheumatology ${ }^{23}$.

US was performed by a rheumatologist fully trained in musculoskeletal ultrasound and with a special interest in scanning enthesitis. Ultrasound was performed using the equipment MyLab (Esaote S.p.A., São PauloBrazil) with a high frequency GS probe $(18 \mathrm{MHz})$. The US evaluation were effected with GS and PD techniques to verify joint ecotextural damage. The $\mathrm{PD}$ specifications have been standardized through of a frequency ranging from 8.0 to $12.5 \mathrm{MHz}$ and pulse repetition frequencies (PRF) ranging from 0.7 to $1.0 \mathrm{kHz}$. In order to quantify the PD signal was used sD, which identify real blood flow and not an artifact.

Nail abnormalities detected by US were graded in both GS and PD techniques. Nail abnormalities seen in GS were semi-quantitatively graded from 0 to 3, as follows: GS0- preserved three-layered nail pattern; GS1light abnormality in the three-layered nail pattern or one sinuosity or nail loss; GS2- intense abnormality in the three-layered nail pattern, still preserving some segments of the nail, more than one sinuosity or nail loss; and GS3- total loss of the three-layered nail pattern, thickening of all of the nail bed with loss of the eco texture or the nail insertion point ([?] $2.5 \mathrm{~mm}$ ) (Figure 1). The nail bed (distance between the ventral plate and the bone margin of the distal phalanx) was measured in both longitudinal (longitudinal mNGS, mm) and transversal planes (transversal mNGS, mm). Nail PD abnormalities (PD0-3) were graded to measure the presence and intensity of nail enthesitis as follows: PD0- absence of the PD signal in any location of the nail bedding; PD1- presence of 1 point or $25 \%$ of the PD signal in any location of the nail bedding, especially at the insertion point of the nail; PD2- presence of 2 to 3 isolated points or $<50 \%$ of the PD signal at any location of the nail bedding, especially at the insertion point of the nail; PD3- presence of PD signal in all of the nail bedding, or $>50 \%$ of the PD signal in all of the nail bedding, especially at the insertion point of the nail (Figure 2). sD was used to measure RI in the nail bed at both longitudinal (longitudinal RI) and transverse plane (transverse RI) (Figure 3). All participants were instructed about the protocol and 
informed consent was given before study procedures.

Statistics

Data are expressed either as frequencies or means (+- standard deviation [SD]) according to the variable. The mean values for sD, GS and PD parameters in PsA patients and their controls were compared by using a two-tailed $t$ test. The Mann-Whitney $\mathrm{U}$ test was used to compare ultrasound semi quantitative scores between PsA patients and controls.

Coefficient of correlation between clinical parameters (age, PASI, disease duration, VASGp and VASNp) and sD, GS and PD data were analyzed using the Pearson and Spearman correlation tests.

The performance of sD measurements to associate with PsA diagnosis and clinical parameters in PsA patients was evaluated by using ROC curve analysis. Statistical analysis was performed using SPSS V.11.5 and p values lower than 0.05 were considered significant.

\section{Results}

A total of 208 nail beds were evaluated using sD. The first, second, third, fourth and fifth nail beds showed abnormalities in $15(7.2 \%), 63(30.3 \%) ; 61(29.3 \%) ; 41(19.7 \%)$; and $28(13.5 \%)$ patients, respectively. Using sD technique, a total of 192 longitudinal and 153 transversal RI measurements were performed. Measurements longitudinal and transversal thickness nail bed number was 120 and 90, respectively.

Nail appearance on GS showed loss of the normal three-layered appearance (GS2 or GS3) in 89 nails (45.9\%).

VASGp was graded 0 (no pain) in 31 individuals of our sample (70.5\%). Accordingly, VASNp or pain in the distal interphalangeal joint was graded 0 (no pain) in the vast majority of the nails evaluated ( $\mathrm{n}=186$, $89.4 \%)$.

Demographic data and clinical parameters of PsA patients and their controls evaluated by US in our sample are shown in Table 1. PsA patients were no older than their controls and showed a trend to be more symptomatic for global pain $(\mathrm{p}=0.07)$ while had significantly more nail pain than their healthy controls and patients with hand OA $(\mathrm{p}=0.004)$. PD signal was detected in 189 nail beds $(92.2 \%)$, showing inflammatory activity in most of the patients and nails examined. Our results for nail US are shown in Table 2. RI measured by sD was significantly lower in PsA patients as compared with their controls in both longitudinal and transverse planes $(\mathrm{p}<0.001)$. In general, GS or PD nail abnormalities were significantly more prevalent in PsA patients as compared with their controls. Accordingly, longitudinal nail bed measurements were significantly longer in PsA patients as compared with healthy controls and hand OA patients. Transverse measurements did not differ significantly between PsA patients and their controls. Significant association was observed between clinical parameters and US findings in our sample. Most of the correlations found were weak to moderate. VASGp for example was moderately correlated with longitudinal RI $(\mathrm{r}=0.322$; $\mathrm{p}=0.037)$. As expected, semiquantitative nail PD scores were significantly correlated with the presence of PD signal $(\mathrm{r}=0.632 ; \mathrm{p}<0.01)$, while semiquantitative nail GS scores were also strongly associated with nail GS $(\mathrm{r}=0.842 ; \mathrm{p}<0.01)$. Interestingly, mNGS transverse measurements were also strongly associated with GS abnormalities and lack of the normal three-layered nail pattern.

\section{Test performance analysis}

The nail inflammatory process was observed when RI values below 0.4, characterizing $100 \%$ sensitivity and $96 \%$ specificity, when the area under the ROC curve was $0.858(\mathrm{p}<0.01)$.

\section{Discussion}

In the present study we have demonstrated that sD measurements are associated with entheseal abnormalities in PsA patients and may help detect inflammatory activity in the management of these patients. GS ultrasound evaluation may demonstrate abnormalities in the shape of the nail bed, loss of the three-layered nail pattern as well as changes in the longitudinal or transversal nail bed in patients with PsA even before clinical defects are present. Using a composite semiquantitative score to GS, we were able to show nail 
plate ultrasonographic abnormalities in PsA patients without clear nail clinical findings. More to the point, test performance analysis demonstrated that RI measurements using $\mathrm{sD}$ in our sample were significantly associated with PsA diagnosis.

Currently, the PD characterize indications important in the joint inflammatory disease. sD parameters have been associated with inflammatory clinical and US changes in spondyloarthritis, as is seen in the literature ${ }^{24}$.

The evaluation of RI allows quantify of the degree of vasodilation of microcirculation. Previous studies have defined that at the joints the value of the RI is usually near 1 in healthy subjects; in case of inflammation this parameter decrease, as synovitis and sacroiliitis cut-off point are $<0.79$ and [?]0.75, respectively, but still there was not a clinical validate defined cut-off value to nail enthesitis, in previous studies ${ }^{25,26}$. There is a potential use of sD to evaluate entheseal involvement in rheumatic diseases, especially when the PD generates doubts about inflammatory tissue activity, as proposed by our study.

Measurements using sD may objectively quantify PD signal in PsA patients, when compered onychomycosis patient ${ }^{27}$. Current study has suggested that measurements detected by nail sD can be associated the atherosclerotic process in PsA patients ${ }^{28}$.

In our study RI measurements in PsA patients were significantly lower than those of the control group (healthy controls and OA patients). That was demonstrated in both longitudinal and transversal measurements and is consistent with findings in a previous study.

It is very interesting to note that $\mathrm{sD}$ changes precede clinical manifestation in PsA patients. In our sample a total of 31 (70.5\%) PsA patients and $186(89.4 \%)$ nails presented sD abnormalities while VASGp and VASNp were 0 , meaning no clinical symptom. These findings suggest that sD measurements in PsA patients may help identify patients with subclinical inflammatory activity.

A systematic review showed studies that characterize PD and sD measurements, but still no other paper had analyzed completely the cut-off point, defining correlations clinical, GS, PD and sD in nail enteses of PsA patients and control groups ${ }^{29}$.

At this point it is also important to point out limitations of the present study. The cross sectional design of our protocol precludes us of establishing a cause-effect association between sD findings and PsA pathophysiology. This study was the non-aleatory selection of the nail bed because of the inexistence of prior references. Further studies including a better description of the sD findings related to nail enthesitis and those of synovial damage in distal interphalangeal joints in both PsA and OA patients will be needed to consolidate our findings.

\section{Conclusions}

In the present study we have demonstrated that nail ultrasound evaluation is a valuable tool to detect inflammatory activity in patients with PsA. sD measurements quantifying inflammatory damage of the entheseal tissue specifically in the nail bed can be used to identify patients with subclinical disease activity and may help in their management. Further studies using a longitudinal design will be needed to evaluate whether the therapeutic management of these subclinical ultrasonographic manifestations will be associated with better outcomes in PsA patients.

\section{List of abbreviations}

GS: Gray scale

OA: Osteoarthritis

PASI: Psoriasis area and severity index

PD: Power Doppler

PsA: Psoriatic arthritis 
RA: Rheumatoid arthritis

RI: resistance index

ROC: Receiver operating curve

sD: spectral Doppler

SD: Standard deviation

US: Ultrasonography

VASGp: Visual analog scale for global pain

VASNp: Visual analog scale for nail pain

mNGS: Nail measured in Gray scale

\section{References}

1. McGonagle D, Conaghan PG, Emery P. Psoriatic arthritis: a unified concept twenty years on. Arthritis and rheumatism $1999 ; 42(6): 1080-1086$.

2. Balint PV, Kane D, Wilson H, McInnes IB, Sturrock RD. Ultrasonography of entheseal insertions in the lower limb in spondyloarthropathy. Annals of the rheumatic diseases 2002 ; 61(10):905-910.

3. McGonagle D, Ash Z, Dickie L, McDermott M, Aydin SZ. The early phase of psoriatic arthritis. Annals of the rheumatic diseases $2011 ; 70$ Suppl 1:i71-76.

4. Leung YY, Tam LS, Kun EW, Li EK. Psoriatic arthritis as a distinct disease entity. Journal of postgraduate medicine 2007 ; 53(1):63-71.

5. Lawry M. Biological therapy and nail psoriasis. Dermatologic therapy 2007 ; 20(1):60-67.

6. McGonagle D, Tan AL, Benjamin M. The biomechanical link between skin and joint disease in psoriasis and psoriatic arthritis: what every dermatologist needs to know. Annals of the rheumatic diseases 2008 ; $67(1): 1-4$

7. Terslev L, Torp-Pedersen S, Savnik A, von der Recke P, Qvistgaard E, Danneskiold-Samsoe B, Bliddal H. Doppler ultrasound and magnetic resonance imaging of synovial inflammation of the hand in rheumatoid arthritis: a comparative study.Arthritis and rheumatism 2003 ; 48(9):2434-2441.

8. Varsamidis K, Varsamidou E, Tjetjis V, Mavropoulos G. Doppler sonography in assessing disease activity in rheumatoid arthritis. Ultrasound in medicine $\mathcal{E}$ biology $2005 ; 31(6): 739-743$.

9. Rubin JM, Adler RS, Fowlkes JB, Spratt S, Pallister JE, Chen JF, Carson PL. Fractional moving blood volume: estimation with power Doppler US. Radiology 1995 ; 197(1):183-190.

10. Stevenson GN, Collins SL, Welsh AW, Impey LW, Noble JA. A Technique for the Estimation of Fractional Moving Blood Volume by Using Three-dimensional Power Doppler US.Radiology 2014 ; https://doi.org/10.1148/radiol.14132363.

11. Naredo E, Moller I, Cruz A, Carmona L, Garrido J. Power Doppler ultrasonographic monitoring of response to anti-tumor necrosis factor therapy in patients with rheumatoid arthritis. Arthritis and rheumatism 2008 ; 58(8):2248-2256.

12. Hammer HB, Kvien TK. Ultrasonography shows significant improvement in wrist and ankle tenosynovitis in rheumatoid arthritis patients treated with adalimumab.Scandinavian journal of rheumatology 2011 ; 40(3):178-182.

13. Fournie B, Margarit-Coll N, Champetier de Ribes TL, Zabraniecki L, Jouan A, Vincent V, Chiavassa H, Sans N, Railhac JJ. Extrasynovial ultrasound abnormalities in the psoriatic finger. Prospective comparative 
power-doppler study versus rheumatoid arthritis. Joint, bone, spine : revue du rhumatisme 2006 ; 73(5):527531.

14. Tang Y, Cheng S, Yang Y, Xiang X, Wang L, Zhang L, Qiu L. Ultrasound assessment in psoriatic arthritis (PsA) and psoriasis vulgaris (non-PsA): which sites are most commonly involved and what features are more important in PsA? Quant Imaging Med Surg 2020; 10(1):86-95.

15. Gutierrez M, Filippucci E, Salaffi F, Di Geso L, Grassi W. Differential diagnosis between rheumatoid arthritis and psoriatic arthritis: the value of ultrasound findings at metacarpophalangeal joints level. Annals of the rheumatic diseases 2011 ; 70(6):1111-1114.

16. Ash ZR, Tinazzi I, Gallego CC, Kwok C, Wilson C, Goodfield M, Gisondi P, Tan AL, Marzo-Ortega $\mathrm{H}$, Emery $\mathrm{P}$ et al. Psoriasis patients with nail disease have a greater magnitude of underlying systemic subclinical enthesopathy than those with normal nails. Annals of the rheumatic diseases 2012 ; 71(4):553556.

17. Mendonca JA, Pansani LN, Mimoto MB, Ferreira ITG, Sanches FB, Pinto TFC, Leandro-Merhi VA, Aquino JLB. Nail enthesis ultrasound and automated software-guided assessment of bilateral common carotid intima-media thickness in psoriasis and psoriatic arthritis: is there a correlation with clinical and laboratory findings? Drugs in Context 2020; 9: 1-2.

18. Ory PA, Gladman DD, Mease PJ. Psoriatic arthritis and imaging. Annals of the rheumatic diseases 2005 ; 64 Suppl 2:ii55-57.

19. van der Heijde D, Sharp J, Wassenberg S, Gladman DD. Psoriatic arthritis imaging: a review of scoring methods. Annals of the rheumatic diseases 2005 ; 64 Suppl 2:ii61-64.

20. Aydin SZ, Ash ZR, Tinazzi I, Castillo-Gallego C, Kwok C, Wilson C, Goodfield M, Gisondi P, Tan AL, Marzo-Ortega $\mathrm{H}$ et al. The link between enthesitis and arthritis in psoriatic arthritis: a switch to a vascular phenotype at insertions may play a role in arthritis development. Annals of the rheumatic diseases 2013 ; 72(6):992-995.

21. Husein El-Ahmed H, Garrido-Pareja F, Ruiz-Carrascosa JC, Naranjo-Sintes R. Vessel resistance to blood flow in the nailfold in patients with psoriasis: a prospective case-control echo Doppler-based study. The British journal of dermatology 2012 ; 166(1):54-58.

22. Taylor W, Gladman D, Helliwell P, Marchesoni A, Mease P, Mielants H. Classification criteria for psoriatic arthritis: development of new criteria from a large international study.Arthritis and rheumatism 2006 ; 54(8):2665-2673.

23. Backhaus M, Burmester GR, Gerber T, Grassi W, Machold KP, Swen WA, Wakefield RJ, Manger B. Guidelines for musculoskeletal ultrasound in rheumatology. Annals of the rheumatic diseases 2001 ; 60(7):641-649.

24. Mendonca JA, Bisetto de Andrade B, Braga de Aquino JL, Leandro-Merhi VA, Damian GB. Spectral Doppler and automated software-guided ultrasound assessment of bilateral common carotid intima-media thickness in spondyloarthritis: is there a correlation with clinical findings? Drugs in Context 2018; 7: 212538.

25. Carotti M, Salaffi F, Morbiducci J, Ciapetti A, Bartolucci L, Gasparini S, Ferraccioli G, Giuseppetti GM, Grassi W. Colour Doppler ultrasonography evaluation of vascularization in the wrist and finger joints in rheumatoid arthritis patients and healthy subjects.European journal of radiology 2012 ; 81(8):1834-1838.

26. Castillo-Gallego C, De Miguel E, Garcia-Arias M, Plasencia C, Lojo-Oliveira L, Martin-Mola E. Color Doppler and spectral Doppler ultrasound detection of active sacroiliitis in spondyloarthritis compared to physical examination as gold standard. Rheumatol Int2017; 37(12):2043-2047.

27. Mendonca JA. Differences of spectral Doppler in psoriatic arthritis and onychomycosis. Revista brasileira de reumatologia 2014 ; 54(6):490-493. 
28. Mendonca JA, Pansani LN, Mimoto MB, et al. Nail enthesis ultrasound and automated software-guided assessment of bilateral common carotid intima-media thickness in psoriasis and psoriatic arthritis: is there a correlation with clinical and laboratory findings?. Drugs Context 2020; 9:2020-1-2.

29. Mendonca JA, Aydin SZ, D'Agostino M. The use of ultrasonography in the diagnosis of nail disease among patients with psoriasis and psoriatic arthritis: a systematic review. Adv Rheumatol 2019 ; 59(1):41; https://doi.org/10.1186/s42358-019-0081-9.

Figure 1. Title: Semiquantitative GS ultrasound score scale for nail abnormalities.

A - GS0; B - GS1; C - GS2 and D - GS3.

Legends: GS0: preserved three-layered nail pattern; GS1: light abnormality in the three-layered nail pattern or one sinuosity or nail loss; GS2: intense abnormality in the three-layered nail pattern, still preserving some segments of the nail, more than one sinuosity or nail loss; and GS3: Total loss of the three-layered nail pattern, thickening of all of the nail bed with loss of the eco texture or the nail insertion point ([?] $2.5 \mathrm{~mm}$ ).

Figure 2. Title: Semiquantitative PD score for nail abnormalities. A - PD0; B - PD1; C - PD2 and D PD3.

Legends: PD1: presence of 1 point or $25 \%$ of the PD signal in any location of the nail bedding, especially at the insertion point of the nail; PD2: presence of 2 to 3 isolated points or $<50 \%$ of the PD signal at any location of the nail bedding, especially at the insertion point of the nail; PD3: presence of PD signal in all of the nail bedding, or $>50 \%$ of the PD signal in all of the nail bedding, especially at the insertion point of the nail.

Figure 3. Title: spectral Doppler measurements.

Legends: A and B: spectral Doppler measurements in a PsA patient. A. Longitudinal spectral Doppler$\mathrm{RI}=0.54$; B. Transversal spectral Doppler $-\mathrm{RI}=0.50$; $\mathrm{C}$ and D: spectral Doppler measurements in a control individual. C. Longitudinal spectral Doppler $-\mathrm{RI}=0.83$ and D. Transversal spectral Doppler $-\mathrm{RI}=0.68$.

\section{Hosted file}

Figure 1.docx available at https://authorea.com/users/338417/articles/471658-highspecificity-of-spectral-and-power-doppler-ultrasonographic-nail-assessment-in-psoriaticarthritis-patients

\section{Hosted file}

Figure 2.docx available at https://authorea.com/users/338417/articles/471658-highspecificity-of-spectral-and-power-doppler-ultrasonographic-nail-assessment-in-psoriaticarthritis-patients

\section{Hosted file}

Figure 3.docx available at https://authorea.com/users/338417/articles/471658-highspecificity-of-spectral-and-power-doppler-ultrasonographic-nail-assessment-in-psoriaticarthritis-patients

\section{Hosted file}

Table 1 to Journal of Evaluation in Clinical Practice.docx available at https://authorea. com/users/338417/articles/471658-high-specificity-of-spectral-and-power-dopplerultrasonographic-nail-assessment-in-psoriatic-arthritis-patients

\section{Hosted file}

Table 2 to Journal of Evaluation in Clinical Practice.docx available at https://authorea. com/users/338417/articles/471658-high-specificity-of-spectral-and-power-doppler- 
ultrasonographic-nail-assessment-in-psoriatic-arthritis-patients 